Brit. Heart f., 1969, 31, 616.

\title{
Reciprocal Rhythm, the Wolff-Parkinson-White Syndrome, and Unidirectional Block
}

\author{
L. SCHAMROTH AND R. L. COSKEY \\ From the University of the Witwatersrand and Baragwanath Hospital, fohannesburg, South Africa, and \\ Cardiopulmonary Laboratory, St. Vincent's Hospital, Los Angeles, California, U.S.A.
}

Reciprocal rhythm can occur with impulses of atrial, AV nodal, or ventricular origin. In reciprocal rhythm with atrial origin, the sinus impulse is conducted anterogradely to the ventricles, but during its passage through the AV node it enters an accessory AV nodal pathway or bypass which enables it to return retrogradely and activate the atria once again (Fig. 1B). Reciprocal return to the atria can only occur through an additional pathway, and the very presence of reciprocal rhythm therefore establishes the existence of such a bypass.

The Wolff-Parkinson-White (WPW) syndrome (1930) is also due to the presence of an AV nodal bypass. In this case, however, the atrial impulse Received February 24, 1969. travels down both the conventional and accessory pathways concomitantly (Fig. 1C) but is conducted at a faster rate down the accessory pathway (pathway $b$ ). It consequently reaches the ventricles prematurely and results in the early inscription of the QRS complex-hence the short $P-R$ interval. Once the rapidly conducted impulse reaches the ventricles, further caudal transmission is not through the specialized conducting tissue, but through ordinary ventricular myocardium-a relatively poor conducting medium. Onward transmission is therefore slower than normal, producing the bizarre and slurred delta wave. The concomitant conduction through the conventional AV nodal pathway (pathway $a$ ) proceeds normally but at a relatively
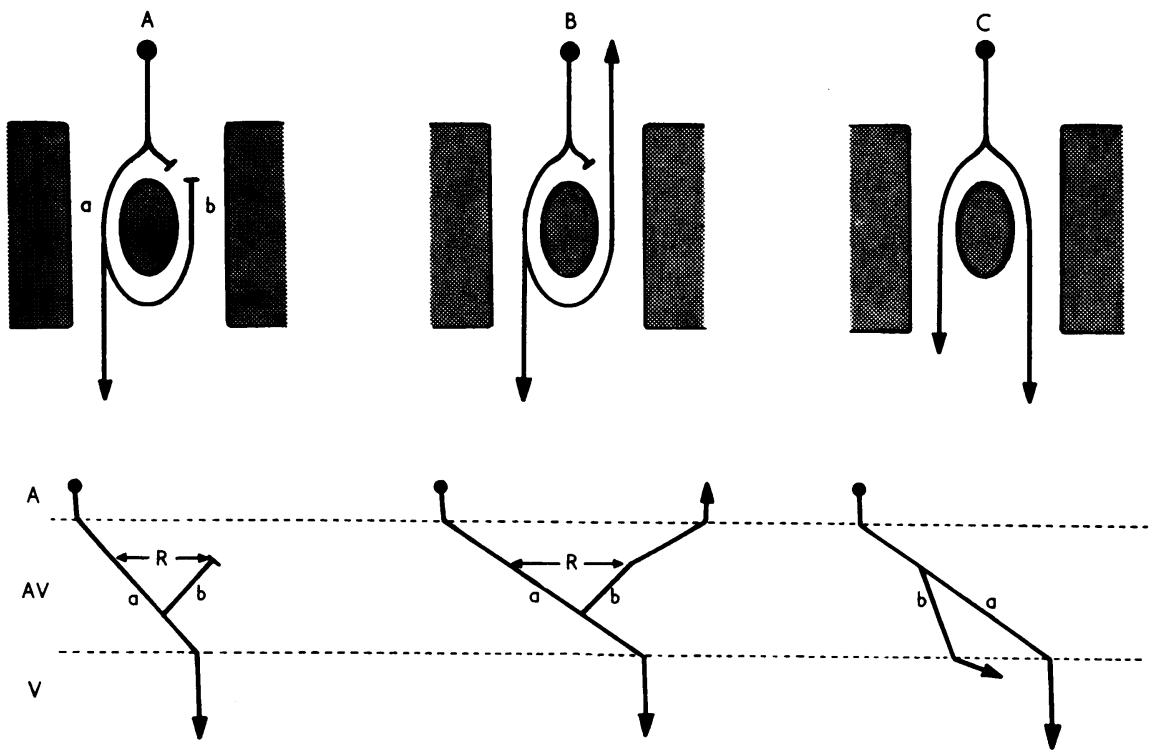

FIG. 1.-Diagrams illustrating the role of an AV nodal bypass in reciprocal rhythm of atrial origin (A and B) and the WPW syndrome (C). See text. $A=$ atrial level. AV $=A V$ nodal level. $V=$ ventricular level. $\mathbf{R}=$ reciprocal time. 
slower rate. Once this impulse reaches the ventricles, however, further onward transmission is through the normal, rapidly conducting, specialized conducting system - the bundle of $\mathrm{His}$ and the bundle-branches. The impulse conducted through this conventional pathway consequently "overtakes" the impulse conducted through the accessory pathway and completes intraventricular conduction in the normal manner. The remainder of the QRS complex is therefore normal in configuration. The typical WPW complex is thus a fusion complex; the initial part-the delta wave-is recorded by the impulse conducted through the accessory pathway, and the normal remainder of the QRS complex is recorded by the impulse conducted through the conventional AV nodal pathway.

The coexistence of reciprocal rhythm and WPW complexes in the same recording shows the intimate relation between these two conditions, their common basis being the presence of a bypass. Very few such cases have been documented. The first example was published by Wolff in 1959 . Schamroth (1960) reported a further case and postulated the intimate relation between these two conditions. Though his case did not at that time manifest WPW complexes, subsequent recordings revealed typical examples. Harris, Semler, and Griswold (1964) reported another case with a similar association, and M. Luria (1968, personal communication) observed two further cases.

The following is another unusual example of this association which is manifest in a case with highgrade and complete AV block. This association, and the presence of retrograde conduction during periods of complete AV block, provided a unique opportunity for the elucidation of the mechanism of unidirectional block.

\section{CASE REport}

The electrocardiograms (Fig. 2 to 4 ) were recorded from a 60-year-old man with generalized vascular insufficiency. Hypertension had been present for many years, the systolic pressure ranging from 190 to $270 \mathrm{~mm}$.
$\mathrm{Hg}$, and the diastolic pressure ranging from 70 to 110 $\mathrm{mm}$. Hg. His ventricular rate usually ranged from $\mathbf{4 0}$ to 42 beats per minute. He had not been taking digitalis for two weeks before the time of the electrocardiographic recordings. Ischaemic gangrene of the right leg had necessitated amputation, and he died one year later of a subarachnoid haemorrhage. Necropsy revealed generalized arteriosclerosis and cardiomegaly (the heart weighed 530 g.). The coronary arteries were "up to 80 per cent narrowed", with atherosclerotic intimal thickening and obvious cholesterol and calcium deposits. There was considerable myocardial fibrosis. Eighteen months before his death he had a documented episode of atrial fibrillation which lasted for 5 days.

Fig. 2 (a continuous recording of standard lead II) shows a basic sinus rhythm at a rate of 81 to 83 a minute (the P-P intervals range from 72 to $74^{\star}$ ). The sinus impulses are conducted with 2:1 AV block. The conducted beats have a prolonged $P-R$ interval which varies from 28 to 39 . When the $P-R$ interval measures 34 or longer, the QRS complex is followed by an inverted $P$ wave-a $\mathbf{P}^{\prime}$ deflection-at an R-P' interval of 21 (6th QRS complex in the first strip; 6th QRS complex in the third strip; the first $4 \mathrm{QRS}$ complexes in the 4th strip). When the $P-R$ interval is shorter than 34, the QRS complex is not followed by a $\mathbf{P}^{\prime}$ deflection.

Occasional bizarre QRS complexes are interspersed throughout the tracing (2nd QRS complex in the first strip; 4th and 6th QRS complexes in the second strip; 3rd QRS complex in the third strip). These bizarre QRS complexes have a slurred proximal limb and are preceded by a $P$ wave at a constant $P-R$ interval of 18 . These bizarre QRS complexes were also observed in other tracings (Fig. 3).

At other times, the basic sinus rhythm was complicated by complete AV block resulting in complete AV dissociation between the sinus rhythm and an AV nodal escape rhythm (Fig. 4 and 5). When, under these circumstances, a $P$ wave fortuitously occurs with a P-R interval of between 35 and 50 , the ensuing QRS complex is followed by a $P^{\prime}$ deflection of the same shape and size as seen during the conducted sinus rhythm (compare with the $\mathrm{P}^{\prime}$ deflection in the tracing of Fig. 2). When the $P$ waves occur with a shorter or longer $P-R$ interval, the $P^{\prime}$ deflection is not manifest. When the preceding $P-R$ interval is within the relatively small range of 47

* All time intervals are expressed in hundredths of a second.

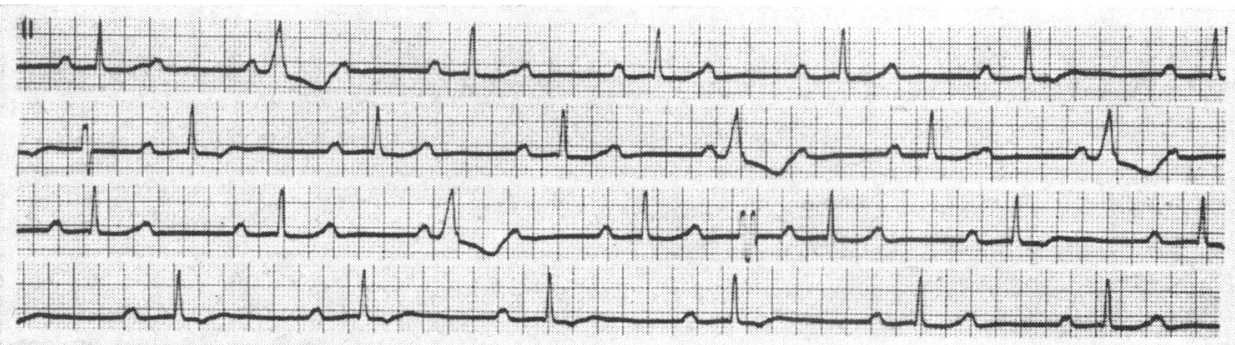

FIG. 2.-Electrocardiogram (continuous recording of standard lead II) showing sinus rhythm with 2:1 AV block, WPW complexes, and atrial echo beats. See text. 


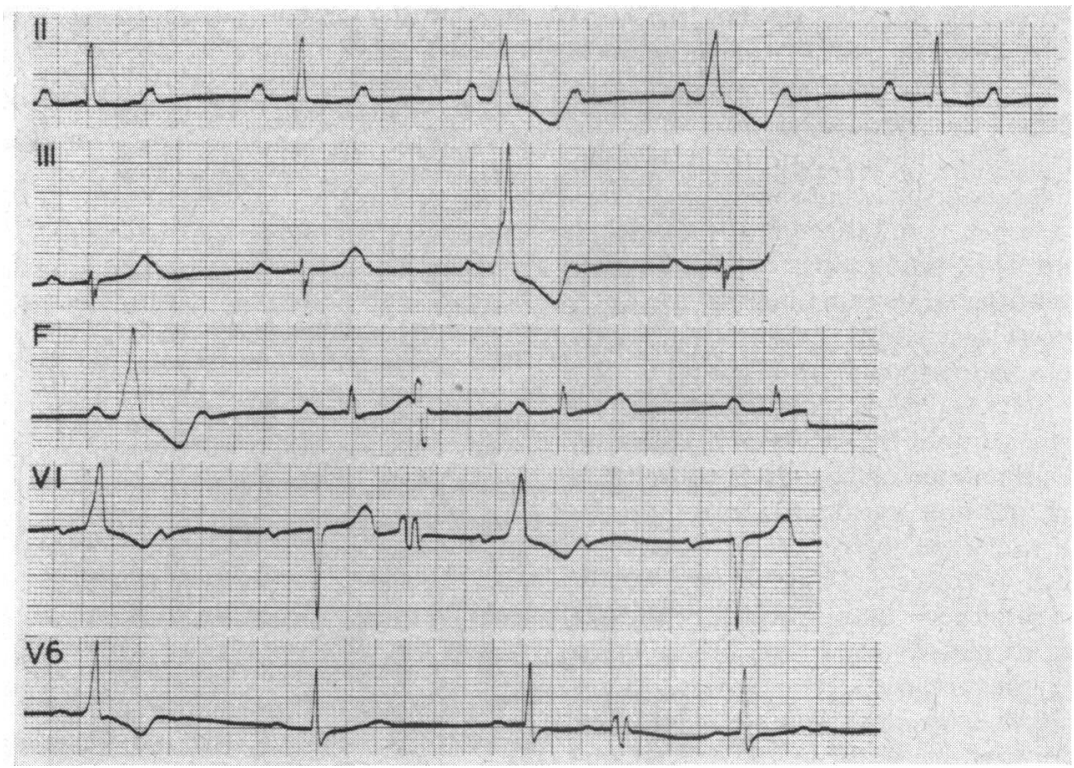

FIG. 3.-Electrocardiograms (leads II, III, aVF, V1, and V6) showing sinus rhythm with 2: 1 AV block and WPW complexes. See text.

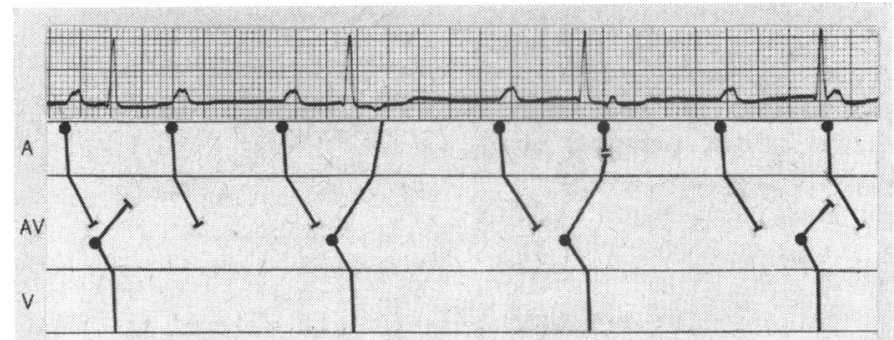

FIG. 4.-Electrocardiogram (standard lead II) showing complete AV block with unidirectional block (anterograde block and retrograde conduction). See text.

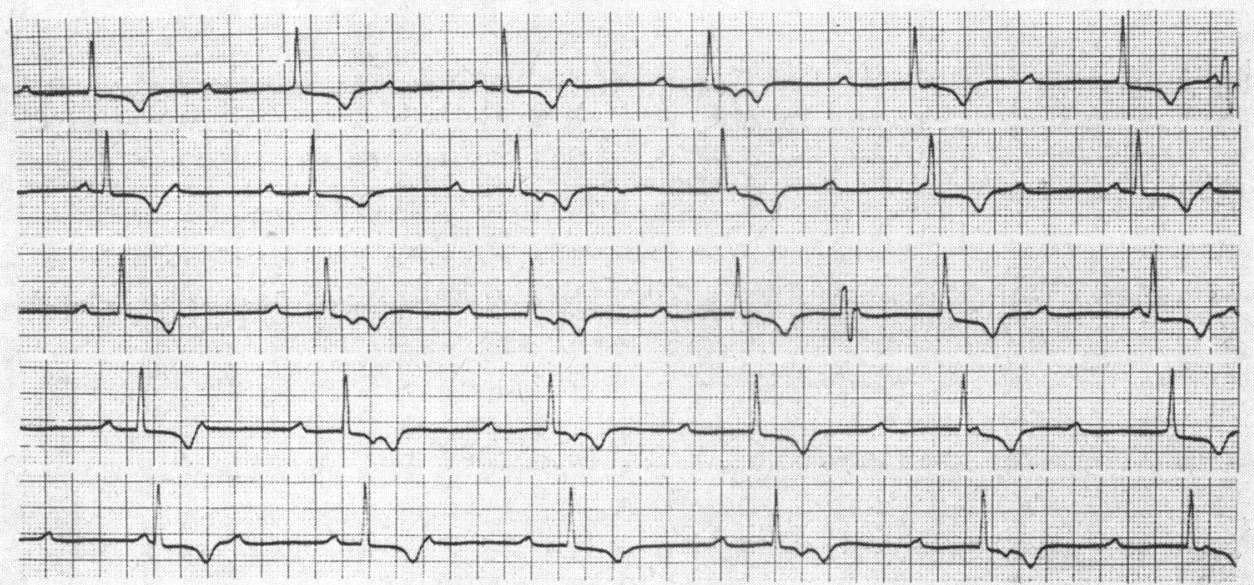

FIG. 5.-Electrocardiogram (continuous recording of standard lead I) showing complete AV block with retrograde VA conduction. See text. 
to 50, the abnormal P wave following the QRS complex is intermediate in shape between the "pure" sinus $P$ wave and the "pure" $P$ ' deflection. These fusion $P$ waves are exemplified by the $P$ wave following the 3rd QRS complex in Fig. 4. This manifestation is also evident in Fig. 5. The $P$ wave following the 4th QRS complex in the 4th strip is almost isoelectric, and thus in between that of the upright sinus $P$ wave and the inverted $\mathbf{P}^{\prime}$ deflection.

\section{Discussion}

\section{The Reciprocal Mechanism}

Reciprocal rhythm with atrial origin is commoner than suggested by the paucity of reported cases. This is probably due to the difficulty in establishing the diagnosis, for it may always be argued that the $\mathbf{P}^{\prime}$ deflection of the presumed reciprocal complex is not in fact a reciprocal beat but a blocked or nonconducted atrial extrasystole. The reciprocal origin of the $\mathbf{P}^{\prime}$ deflection can only be proved by the following rigid and rarely observed criteria (Schamroth and Yoshonis, 1969).

(1) Prolonged Reciprocal Time. The $\mathrm{P}^{\prime}$ deflection only occurs with a relatively long reciprocal time, as may be reflected by a long preceding $P-R$ interval. Such selective linkage would be very fortuitous in the case of an atrial extrasystole. This is well shown in this case. During periods of 2:1 AV block, as well as during periods of complete AV block, the $\mathrm{P}^{\prime}$ deflection is only recorded when preceded by a long $P-R$ interval. The long $P-R$ inter$\mathrm{val}$ is necessary to permit recovery of the atria-or an upper common AV nodal pathway (see below)before the arrival of the reciprocal impulse. This is shown in Fig. 1A and B. The bypass is usually situated in the middle of the AV node. The two pathways join to form both upper and lower common pathways. This is probably an oversimplification, as several pathways may in fact be present. The sinus impulse enters the upper common pathway and is then conducted down the conventional pathway (pathway a), but its transmission down the bypass is blocked as a result of refractoriness, i.e. there is a difference in refractoriness between the conventional and accessory pathways. When the impulse reaches the lower common pathway (through pathway $a$ ) it is able to enter the lower end of the bypass (pathway $b$ ), and is then conducted retrogradely through this pathway. The basic requirements for reciprocal rhythm are therefore an AV nodal bypass, with unidirectional block (anterograde block with retrograde conduction) in the bypass. If reciprocal time is relatively short (as illustrated by the interval labelled $R$ in Fig. 1A), the returning impulse finds the upper common pathway still in a refractory state and is consequently blocked. If reciprocal time is relatively long (as illustrated by the interval $R$ in Fig. 1B), the returning impulse encounters a fully recovered and responsive upper common pathway, and is consequently conducted through to the atria to inscribe the $\mathbf{P}^{\prime}$ deflection.

Though the long reciprocal time is usually due to a prolongation of anterograde conduction-as reflected by a long $P-R$ interval-it may occasionally be due to a prolonged retrograde time as a result of delay in conduction through the bypass (Schamroth and Yoshonis, 1969). While it may be argued that the required delay is necessary to allow the atria themselves to recover, this appears to be unlikely since the refractory period of the atria is relatively short, shorter than the refractory period of the AV nodal pathway and shorter than the usually documented reciprocal times. It seems more likely that a long reciprocal time is necessary to allow for the recovery of an upper AV nodal pathway which has a longer refractory period than the atria. The existence of such an upper common AV nodal pathway has also been deduced from other criteria (Schamroth and Yoshonis, 1969).

(2) Identical $P^{\prime}$ Deflection with $A V$ Nodal or Ventricular Impulses. The diagnosis of reciprocal rhythm with atrial origin is further substantiated by the presence, in recordings from the same patient, of AV nodal beats with retrograde conduction, which results in $\mathrm{P}^{\prime}$ deflections of the same shape and size as the $\mathbf{P}^{\prime}$ deflection seen during the suspected reciprocal sequences. Similar "retrograde" $P$ ' deflections may also occur with ventricular impulses. As the $\mathbf{P}^{\prime}$ deflection during $\mathrm{AV}$ nodal or ventricular rhythm is the result of retrograde activation of the atria, the identical $P^{\prime}$ deflection during the suspected reciprocal rhythm must also be due to retrograde activation, and not the result of an atrial extrasystole.

This is clearly shown in this case. The $P^{\prime}$ deflection due to retrograde conduction of the AV nodal impulses during the periods of complete AV block is identical to the $\mathrm{P}^{\prime}$ deflection seen in the suspected reciprocal sequences.

(3) Variation of Coupling Interval with Fixed $R-P^{\prime}$ Time. Though the two aforementioned criteria are the most important factors upon which the diagnosis of reciprocal rhythm with atrial origin is based, additional evidence was present in this case. This was the variation of the coupling interval with fixed $R-P^{\prime}$ time.

The coupling interval is the interval between an 
ectopic beat and its preceding beat. The coupling intervals of ventricular extrasystoles are usually constant. The coupling intervals of atrial extrasystoles-the interval between the $P^{\prime}$ deflection of the extrasystole and the preceding $P$ wave-also tend to be constant but their variation is greater than that seen with ventricular extrasystole. The variation of the $\mathbf{P}-\mathrm{P}^{\prime}$ interval in this case ranges from 56 to 62 (Fig. 2), and despite this wide variation, the $\mathrm{R}-\mathrm{P}^{\prime}$ interval in any one recording remains constant (e.g. 22 in Fig. 2). It would indeed be a remarkable coincidence if, under these circumstances, the $\mathbf{P}^{\prime}$ deflection represented the discharge of an atrial extrasystolic focus where the resulting variation of the coupling intervals was such that the R-P' always and fortuitously remained constant.

The reciprocal origin of the $\mathbf{P}^{\prime}$ deflection is further substantiated by the presence of atrial fusion complexes, resulting from the concomitant invasion of the atria by the sinus and AV nodal impulses, each activating part of the atria and resulting in a $P$ wave whose shape is in between that of the "pure" sinus $P$ wave and the "pure" AV nodal $P^{\prime}$ deflection (Fig. 4). Atrial fusion complexes will occur when the coupling interval equals the $\mathrm{P}-\mathrm{P}$ of normal sinus interval. In other words, $\mathbf{P}-\mathbf{R}+\mathbf{R}-\mathbf{P}^{\prime}$ must be equal to the P-P interval. This is evident in Fig. 5. The P-P intervals measure 68 to 72 ; the $R-P^{\prime}$ intervals measure 21 ; and whenever the $P$ wave occurs with a $P-R$ interval of 47 to 51 , an atrial fusion complex ensues, e.g. $49+21=70$.

It should also be added that for the diagnosis of reciprocal rhythm with atrial origin, the $\mathbf{P}^{\prime}$ deflection must have all the features of a "retrograde" $P$ ' deflection, i.e. it must be negative in leads II, III, and aVF, with a frontal plane axis in the region of minus 90 degrees. This was evident in this case where the frontal plane $P^{\prime}$ axis was situated at minus 95 degrees (not illustrated).

\section{The WPW Mechanism}

The bizarre QRS complexes, at first glance, seem to be ventricular escape beats. Closer analysis, however, reveals that they are not. The interval between the bizarre QRS complex and the preceding QRS complex measures 140 and is therefore shorter than the conducted sinus interval of 150 . If this were an escape interval it should anticipate every conducted sinus interval, i.e. the escape should occur every time and not just occasionally. Furthermore, this "escape" interval is not constant but its variation is nevertheless such that the $P-R$ interval preceding every bizarre $Q R S$ complex is always constant. This, too, would be a remarkable coincidence. This is also evident when the bizarre QRS complexes occur consecutively. When this occurs, the $R-R$ intervals vary considerably, e.g the consecutive intervals between the bizarre $Q R S$ complexes in standard lead II of Fig. 3 measure 134 and 142, respectively. And despite this variation, the constancy of the P-R interval is maintained at 18 , thereby indicating the causal relation between the bizarre $Q R S$ complex and its preceding $P$ wave. These bizarre QRS complexes therefore represent sinus impulses with abnormal intraventricular conduction. They do not, however, conform to the criteria of phasic aberrant ventricular conductionthe temporary or intermittent abnormal intraventricular conduction of a supraventricular impulse-a phenomenon resulting from difference in refractoriness in the bundle-branches. In this condition there is no abbreviation of the P-R interval with the abnormal intraventricular conduction. The abnormal intraventricular conduction resulting in the bizarre QRS complexes must therefore be due to the presence of a bypass which facilitates rapid transmission of the supraventricular impulse to the ventricular myocardium, as occurs in the WPW syndrome. This is also reflected by the configuration of the QRS complexes, the initial characteristic slurring being evident in all of the bizarre QRS complexes.

The WPW syndrome is usually associated with a short $P-R$ interval, but clear examples with long or relatively long $P-R$ intervals have been reported (Ohnell, 1944; Wolff and White, 1948; Glushien and Goldblum, 1950). This case shows that it is not the short P-R interval as such that is of necessity the significant feature of the WPW syndrome but rather a shortening of the $P-R$ interval. Thus, while the $P-R$ interval associated with the bizarre QRS complexes in this case is shorter than that associated with the normally conducted impulses, it is nevertheless still well within normal limits.

The association of reciprocal rhythm of atrial origin and the WPW syndrome in the same tracing emphasizes the common basic mechanism of these two conditions, viz. the presence of reciprocal pathway or bypass. The particular condition which becomes manifest is dependent on a critical variation in refractoriness or relative refractoriness of the accessory pathway. When both pathways are equally responsive, the impulse is conducted anterogradely down both pathways concomitantly, and the WPW complex is recorded (Fig. 1C). When anterograde conduction through the conventional pathway is possible but is blocked through the bypass, the reciprocal mechanism occurs. The reciprocal mechanism may be complete (Fig. 1B), or it may be associated with concealed conduction in an upper common pathway (Fig. 1A). 
A close relation between reciprocal rhythm of atrial origin, the WPW syndrome, and atrial fibrillation has also been postulated (Schamroth and Krikler, 1967). The reciprocal pathway facilitates a relatively rapid return of the sinus impulse to the atria which may stimulate the atria during their terminal simple out-of-phase state-the vulnerable period-and consequently precipitate atrial fibrillation. The documented episode of atrial fibrillation in this patient was probably due to this mechanism.

\section{Significance of a Reciprocal Pathway in Unidirectional Block}

The electrocardiograms in Fig. 4 and 5 show complete AV block between sinus rhythm and an AV nodal escape rhythm. There is occasional retrograde conduction of the AV nodal impulse, and this is related to a critical preceding $P-R$ interval. This form of unidirectional block has been well documented and has been extensively reviewed by Scherf and Cohen (1964). Its precise mechanism, however, is debatable and subject to controversy. Some authors have assumed a functional longitudinal dissociation in the AV node with one functional pathway permitting retrograde conduction. Others have postulated the existence of an accessory anatomical pathway which permits retrograde conduction to the atria. Scherf, Cohen, and Orphanos (1964), however, question this postulate, for they argue that it seems strange that the accessory pathway should only be used for retro- grade conduction and not for anterograde conduction. Nevertheless, this would appear to be precisely the situation that occurs, as is clearly shown in this case by the fortuitous association of reciprocal rhythm, the WPW syndrome, and unidirectional AV block. This association indicates the following sequence of events.

The sinus impulse enters the upper common AV nodal pathway (Fig. 6A), but its entry into the bypass (pathway $b$ ) is blocked due to a longer period of refractoriness of this pathway as compared to the refractoriness of the conventional pathway (pathway $a$ ). It therefore enters the conventional pathway but is blocked in the lower regions of this pathway, proximal to the lower AV nodal common pathway, due to the presence of true AV blockan absolute increase in refractoriness. The AV nodal regions that have just been activated undergo the usual period of refractoriness (Fig. 6B), and any AV nodal impulse which occurs at this time will find both pathway $a$ and the upper common pathway (via pathway $b$ ) blocked. An AV nodal impulse that occurs slightly later (Fig. 6C), however, may find the lower regions of pathway $a$ still refractory but the upper common pathway recovered and responsive; its conduction to the atria then becomes possible.

It seems, therefore, that unidirectional block (anterograde block and retrograde conduction) in cases of complete AV block is due to the presence of (1) an AV nodal bypass, (2) differential refractoriness of this bypass from the conventional AV nodal

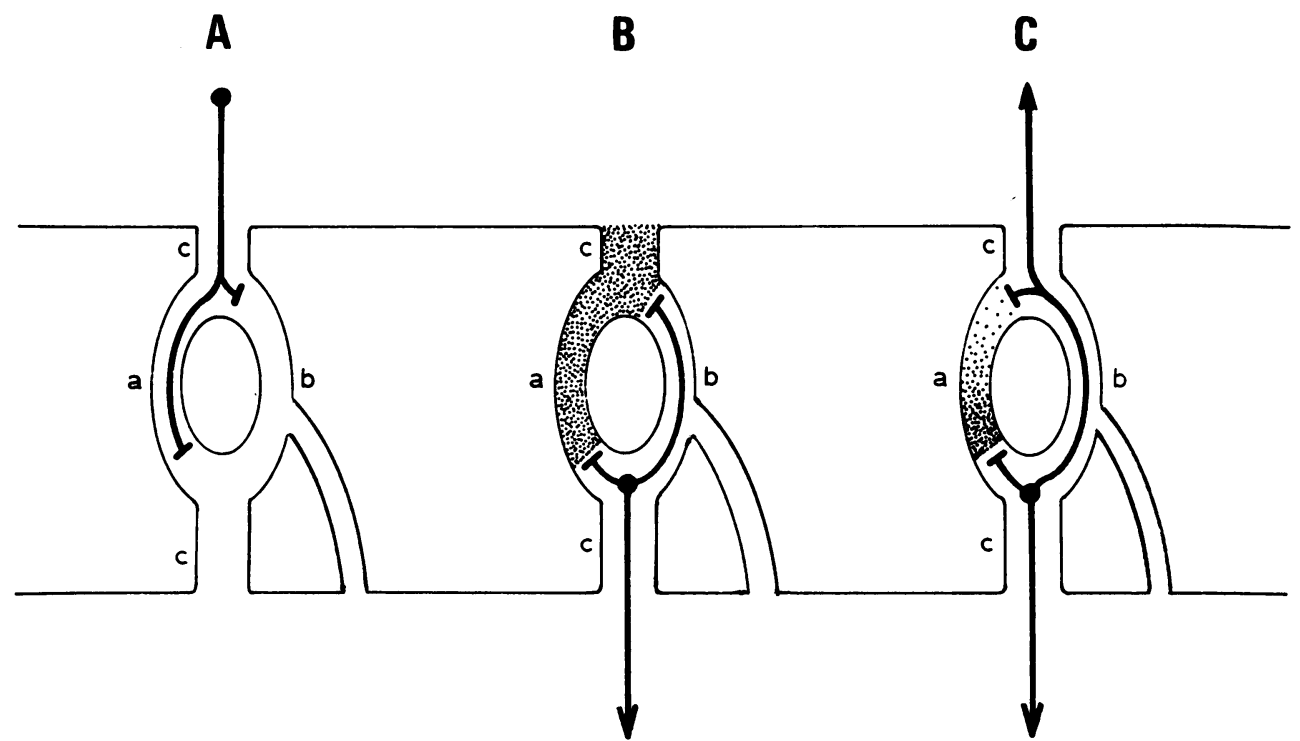

Fig. 6.-Diagrams illustrating the mechanism of unidirectional AV block in the presence of an AV nodal bypass. See text. 
pathway, and (3) differential refractoriness of different AV nodal levels. The bypass allows retrograde conduction with critical recovery times of an upper common pathway.

This sequence of events is further evidence of the critical mechanism of AV transmission. It is well known that layers of varying refractoriness within the AV node may give rise to complex conduction disturbances. It is evident in the phenomenon of concealed conduction, where partial or incomplete antero- or retrograde penetration into the AV node results from different states of horizontal refractoriness within the AV node. And this partial penetration may itself disturb the refractoriness of the different AV nodal levels and create further complex differential refractoriness. This principle is clear from the simplest form of concealed conduction-the interpolated ventricular extrasystoles-where partial retrograde penetration of the extrasystolic impulse indicates the presence of at least two layers of differential horizontal refractoriness within the AV node. It is not only horizontal differential refractoriness that may cause these complex AV transmission disturbances, for longitudinal differential refractoriness between different AV conduction pathways may also contribute to the complexity of AV conduction disturbances. A combination of differential horizontal and longitudinal refractoriness has been invoked to explain the paradox of the supernormal phase of AV conduction (Moe, Childers, and Merideth, 1968), as well as a paradoxical temporal gap in AV conduction (Durrer, 1968). This case is further evidence of this phenomenon. It reflects the complex nature of AV transmission, the significant role of accessory AV pathways, and the intimate relation of different AV conduction manifestations.

\section{SUMMARY}

A case of reciprocal rhythm with atrial origin is reported. This coexisted with the WPW syndrome. The rhythm alternated with periods of complete AV block showing retrograde ventriculoatrial conduction. These associations reflect the significant role of an AV nodal bypass in the genesis of many conduction disturbances, the bypass being common to the mechanisms of reciprocal rhythm, the WPW syndrome, and unidirectional AV block.

We wish to express our thanks to the Photographic Department, Department of Medicine, University of the Witwatersrand, for the photographic reproductions.

\section{REFERENCES}

Durrer, D. (1968). Electrical aspects of human cardiac activity: A clinical-physiological approach to excitation and stimulation. Cardiovasc. Res., 2, 1.

Glushien, A. S., and Goldblum, H. L. (1950). Aberrant atrioventricular conduction with normal P-R interval and prolonged QRS complex simulating bundle-branch block. Amer. Heart f., 40, 476.

Harris, W. E., Semler, H. J., and Griswold, H. E. (1964). Reversed reciprocating paroxysmal tachycardia controlled by guanethidine in a case of Wolff-ParkinsonWhite syndrome. Amer. Heart $\mathcal{F}$., 67, 812.

Moe, G. K., Childers, R. W., and Merideth, J. (1968). An appraisal of "supernormal" AV conduction. Circulation, 38, 5 .

Öhnell, R. F. (1944). Pre-excitation, a cardiac abnormality. Acta med. scand., Suppl. 152.

Schamroth, L. (1960). Reversed reciprocating paroxysmal tachycardia and its relationship to the Wolff-ParkinsonWhite syndrome. Amer. Heart f., 59, 506.

- and Krikler, D. M. (1967). The problem of lone atrial fibrillation. S. Afr. med. F., 41, 502.

- , and Yoshonis, K. (1969). Mechanisms in reciprocal rhythm. Amer. F. Cardiol. In the press.

Scherf, D., and Cohen, J. (1964). The Atrioventricular Node and Selected Cardiac Arrhythmias, p. 208. Grune and Stratton, New York.

$\longrightarrow,-$ and Orphanos, R. P. (1964). Retrograde activation of atria in atrioventricular block. Amer. $\mathcal{F}$. Cardiol., 13, 219.

Wolff, L. (1959). Anomalous atrioventricular excitation (Wolff-Parkinson-White syndrome). Circulation, 19, 14.

-, Parkinson, J., and White, P. D. (1930). Bundlebranch block with short $P-R$ interval in healthy young people prone to paroxysmal tachycardia. Amer. Heart f., 5, 685.

-, and White, P. D. (1948). Syndrome of short P-R interval with abnormal $Q R S$ complexes and paroxysmal tachycardia. Arch. intern. Med., 82, 446. 